Supporting Information for:

\title{
Non-Covalent Coatings on Carbon Nanotubes Mediate Photosensitizer Interactions
}

Christopher P. Horoszko ${ }^{\dagger}$, Peter J. Schnatz ${ }^{\S}$, Januka Budhathoki-Upretyo, Rahul V. RaoPothuraju $^{\phi}$, Ronald L. Koder ${ }^{\S}$, Daniel A. Heller ${ }^{\dagger *}$

$\dagger$ Weill Cornell Medicine, Cornell University, New York, NY 10065, United States

$\$$ Memorial Sloan Kettering Cancer Center, New York, NY 10065, United States

$\S$ Department of Physics, City College of New York, New York, NY 10031, United States

${ }^{\circ}$ Department of Textile Engineering, Chemistry and Science, North Carolina State University, Raleigh, NC 27606, United States

${ }^{\star}$ College of Arts and Sciences, New York University, New York, NY 10003, United States

" Graduate Programs of Physics, Chemistry, Biochemistry and Biology, The Graduate Center of CUNY, New York, New York 10016, United States

*E-mail: hellerd@mskcc.org 
Cationic polycarbodiimide (amine)

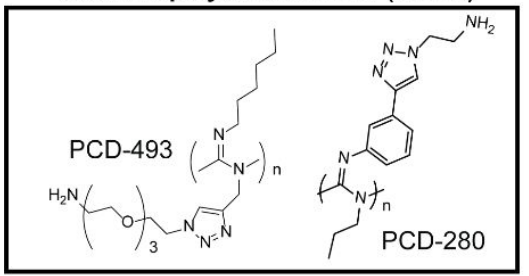

Neutral polycarbodiimide (PEG/alcohol)

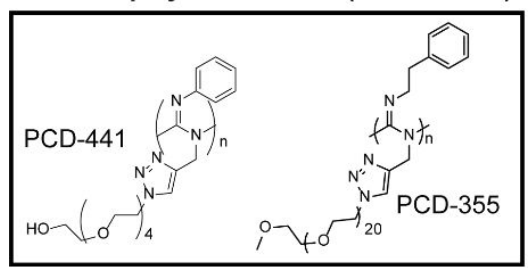

SsDNA

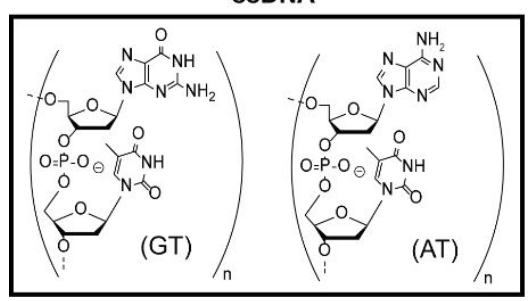

Anionic polycarbodiimide (carboxylate)

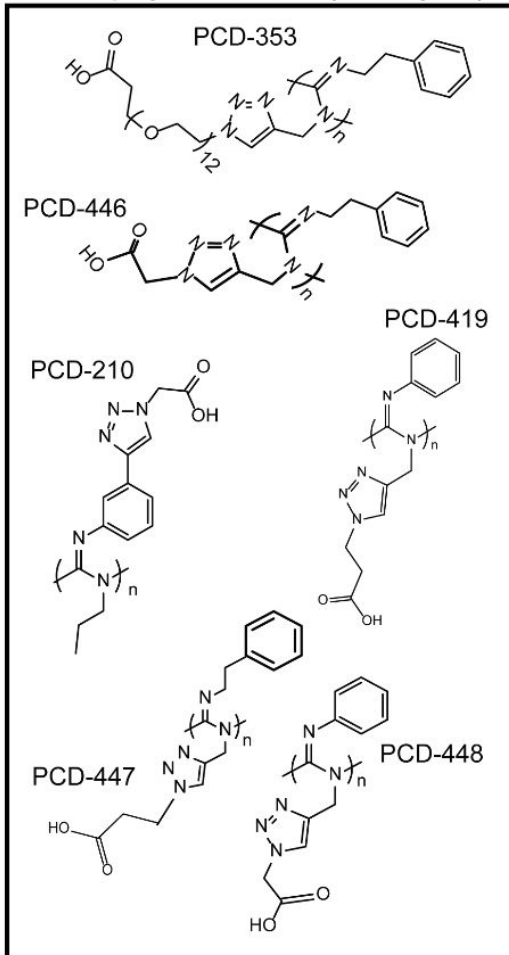

Surfactant

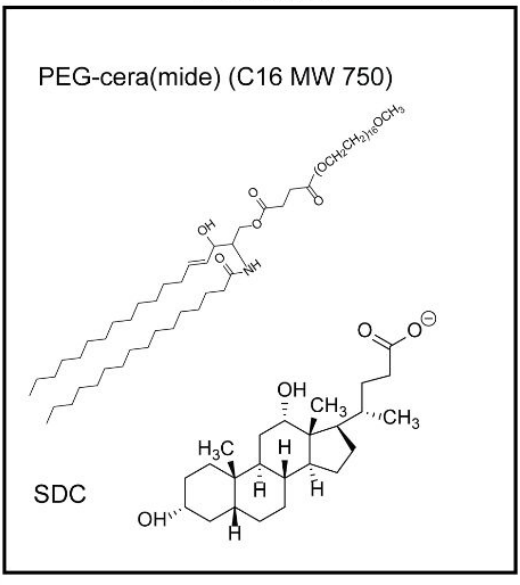

Carbohydrate polymer (carboxylate)

$\mathrm{CMC}$

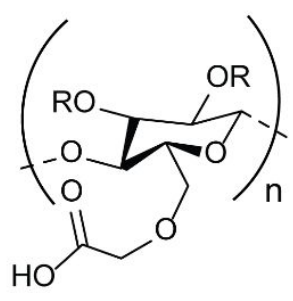

Figure S1 Encapsulation and polymers used to suspend SWCNTs. Chemical structures of the coatings used to suspend the CoMoCat or HiPco-produced CNTs. 

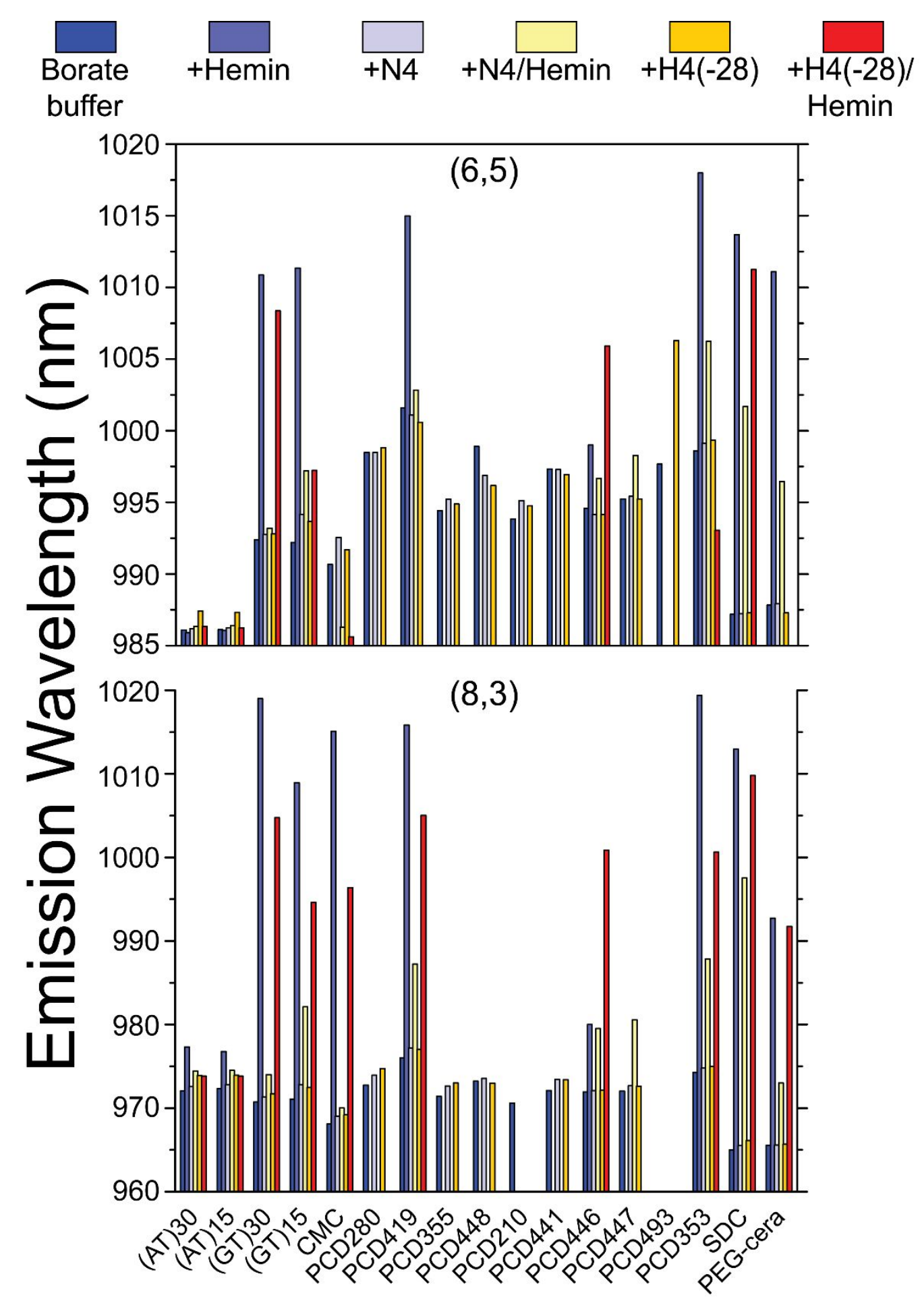

Figure S2 Coating-mediated CNT emission response to hemin/protein-bound hemin. CNT emission center wavelength response to hemin, protein-bound hemin, and controls, reported as a function of the CNT encapsulation/coating for the narrow diameter chiralities $(6,5-$ top; 8,3 - bottom). $1 \mu \mathrm{g} / \mathrm{mL}$ coated-CNT, $1 \mu \mathrm{M}$ each of porphyrin/protein were added. PCD - polycarbodiimide; SDC - sodium deoxycholate; PEG - polyethylene glycol; cera ceramide; (AT)n/(GT)n - ssDNA polymer of n-repeats; N4/H4(-28) - hemin binding proteins. 

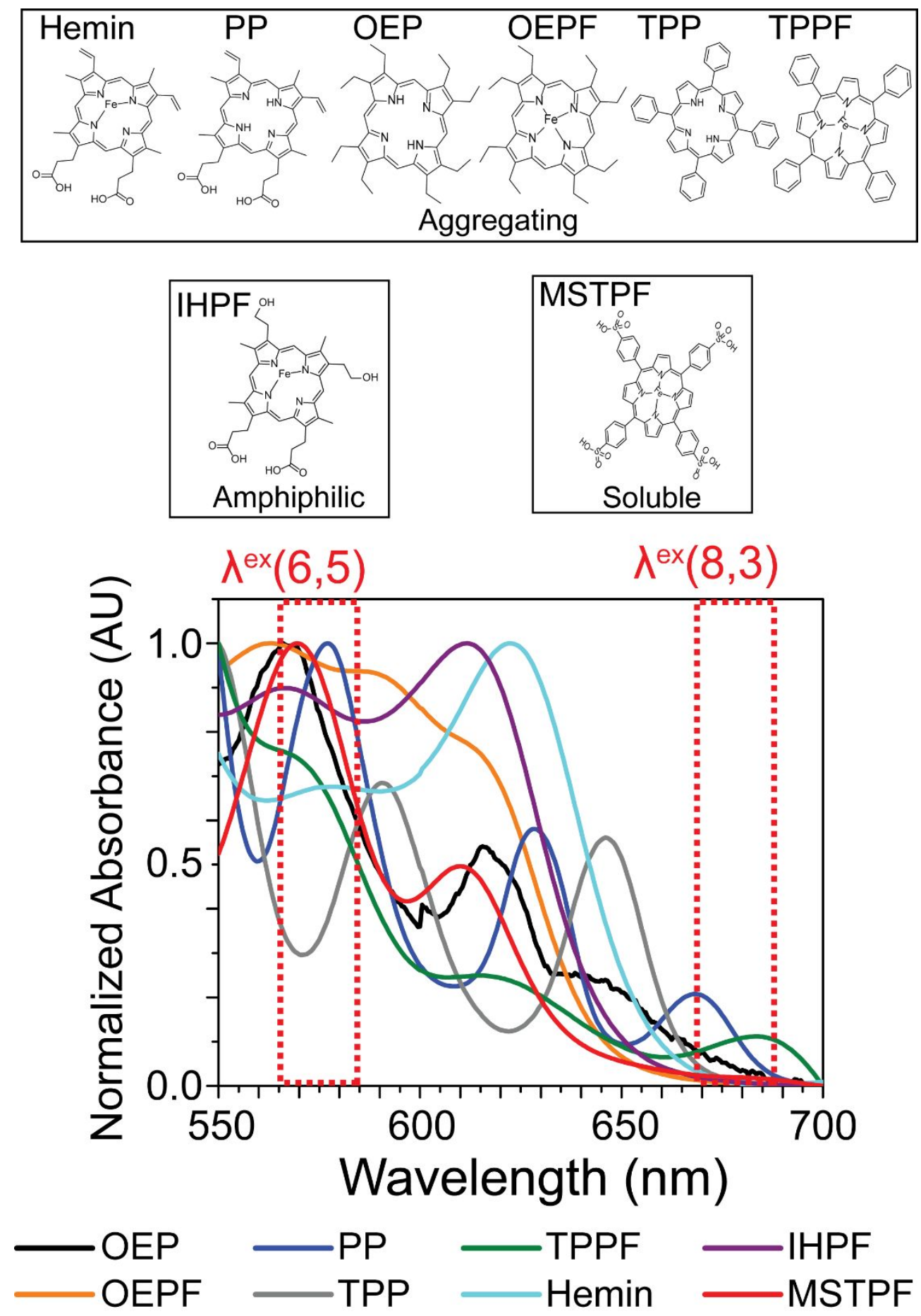

Figure S3 Porphyrin species and Q-bands. Top - chemical structures of the porphyrin analogs used in this work. Analogs are grouped into three categories depending on their solubility in an aqueous environment. Bottom - absorption spectrum overlay of the porphyrin Q-bands. The continuous wave excitation laser (+bandwidth) used in the CNT acceptor assay are outlined by red dashed boxes. 
(a)

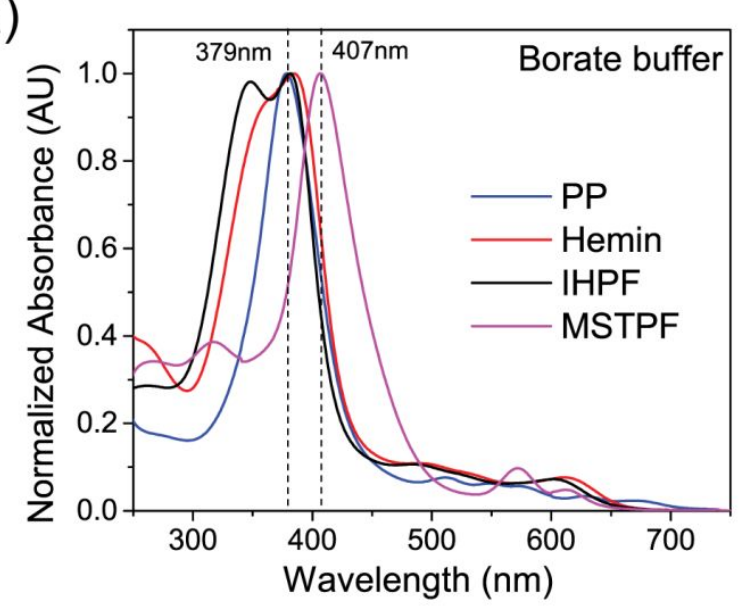

(b)

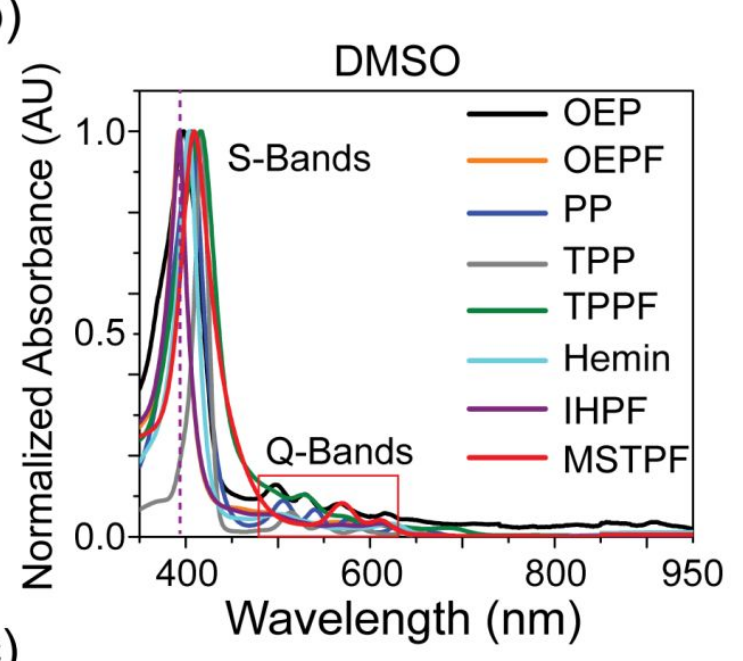

(c)

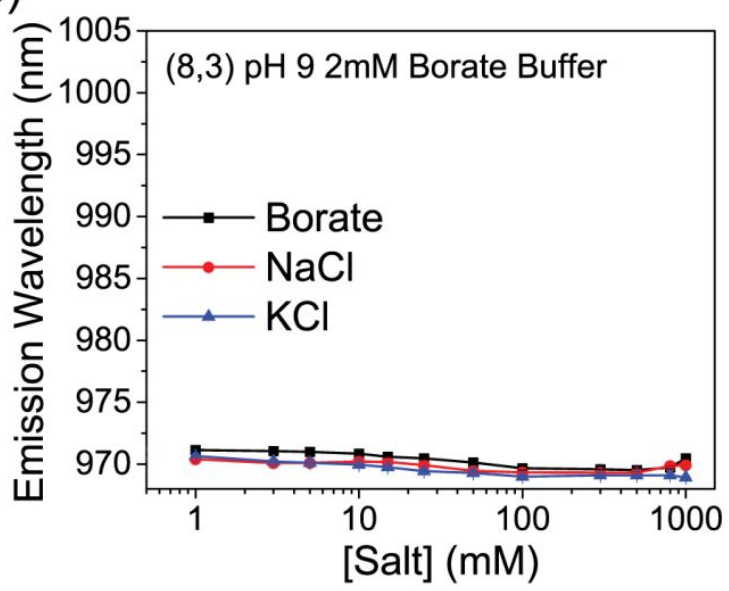

Figure S4 Porphyrin spectral bands in solvents, and background buffer effect. (a)

Sspectrophotometry of aqueous solutions containing $15 \mu \mathrm{M}$ porphyrins. The center wavelengths of two optical transitions are outlined. (b) Spectrophotometry of DMSO solutions containing $15 \mu \mathrm{M}$ porphyrins. The Soret (S-band) and Q-band regions are outlined. The dashed line is a visual benchmark of the highest energy Soret band for the listed porphyrins in DMSO. (c) Excitationemission spectroscopy of the $(8,3) 446-\mathrm{CNT}$ as a function of aqueous buffer component concentration. The starting aqueous solution (not shown, $0 \mathrm{mM} \sim 971 \mathrm{~nm}$ emission) was buffered by Borate as indicated. 


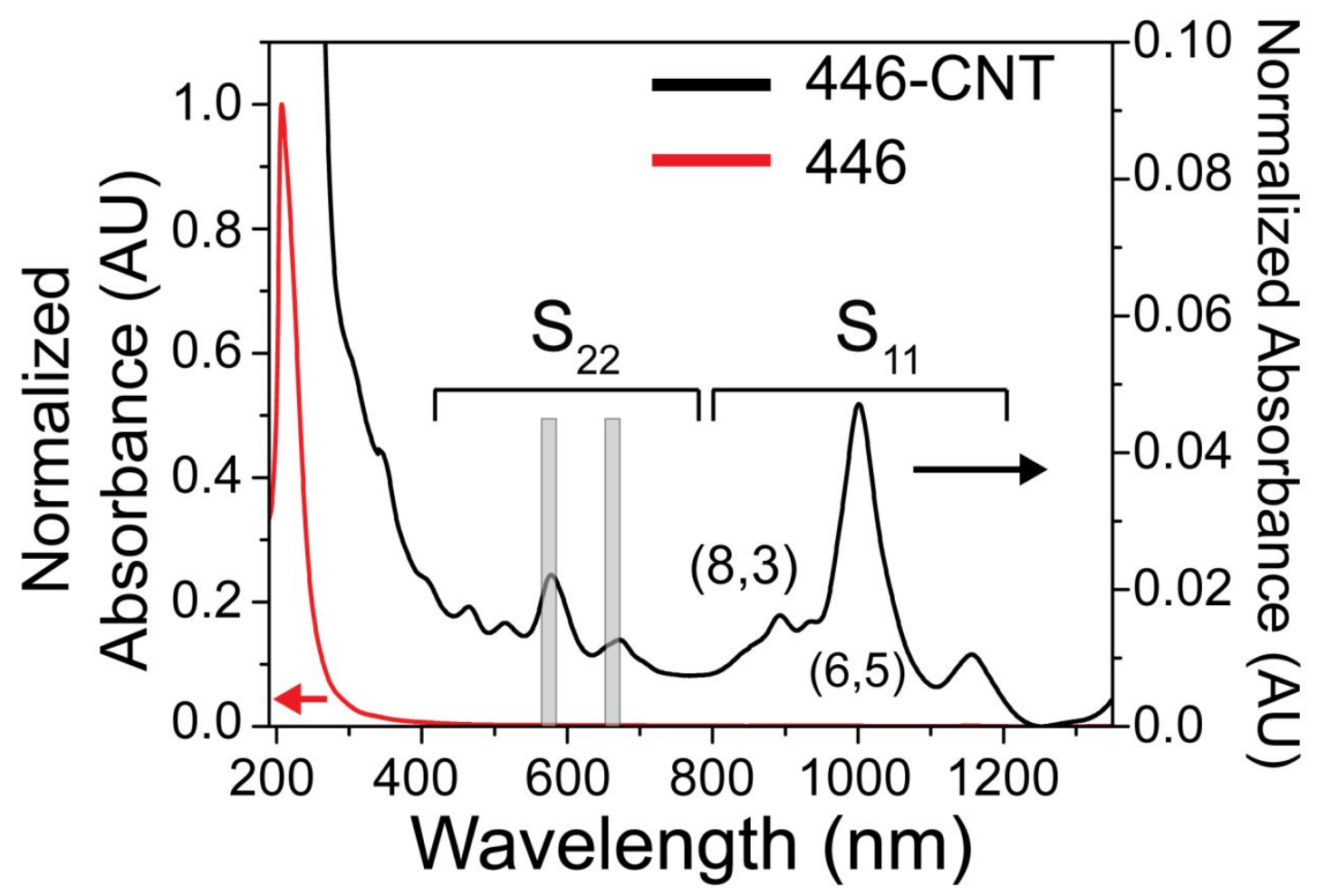

Figure S5 UV-Visible-NIR spectra of the polymer-nanotube complex. Absorbance spectra of the polycarbodiimide polymer $446(1 \mu \mathrm{M}$, aqueous $)$ and 446-CNT complexes (1 $\mu \mathrm{g}$, aqueous). The CNT optical transitions $\left(\mathrm{S}_{22} / \mathrm{S}_{11}\right)$ are denoted. Gray bars outline the excitation laser bandwidths. 

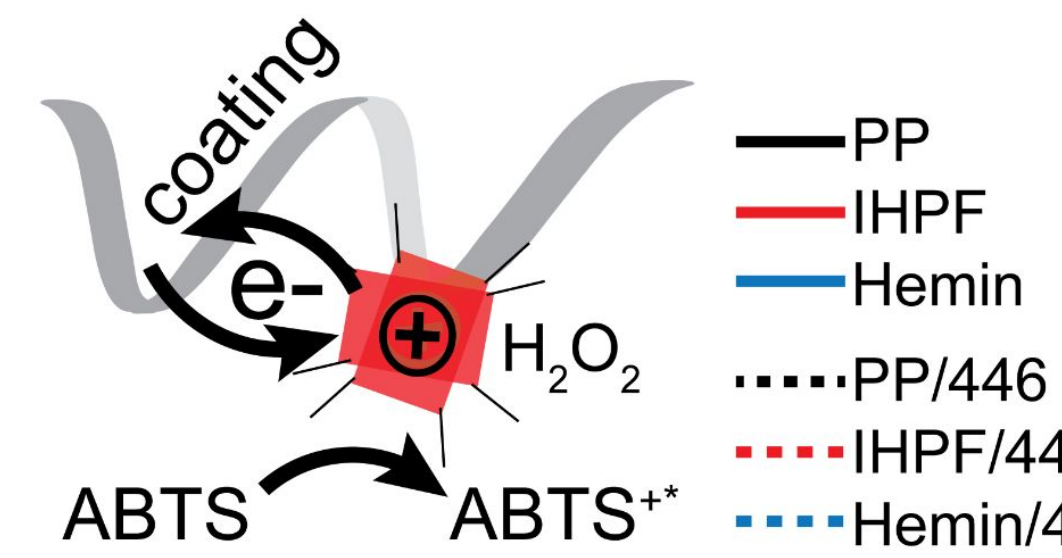

$-=-$ IHPF/446

- - - - Hemin/446

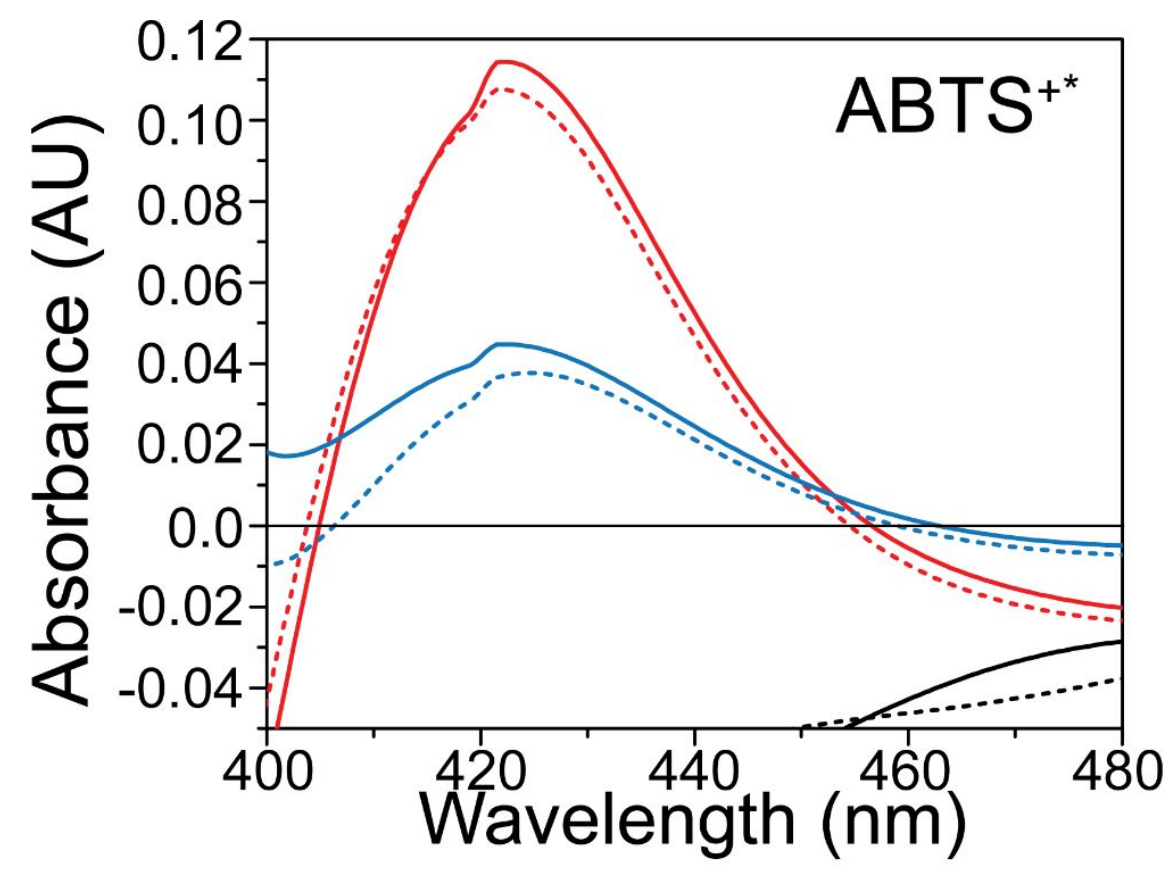

Figure S6 ABTS redox sensitization assay of polymer-porphyrin complexes. 15 $\mu \mathrm{M}$ of each porphyrin was mixed with assay reagents in the presence/absence of $6 \mu \mathrm{g}$ of polymer 446 in aqueous buffer. Absorption was measured in the ABTS radical window after hydrogen peroxide substrate addition. 


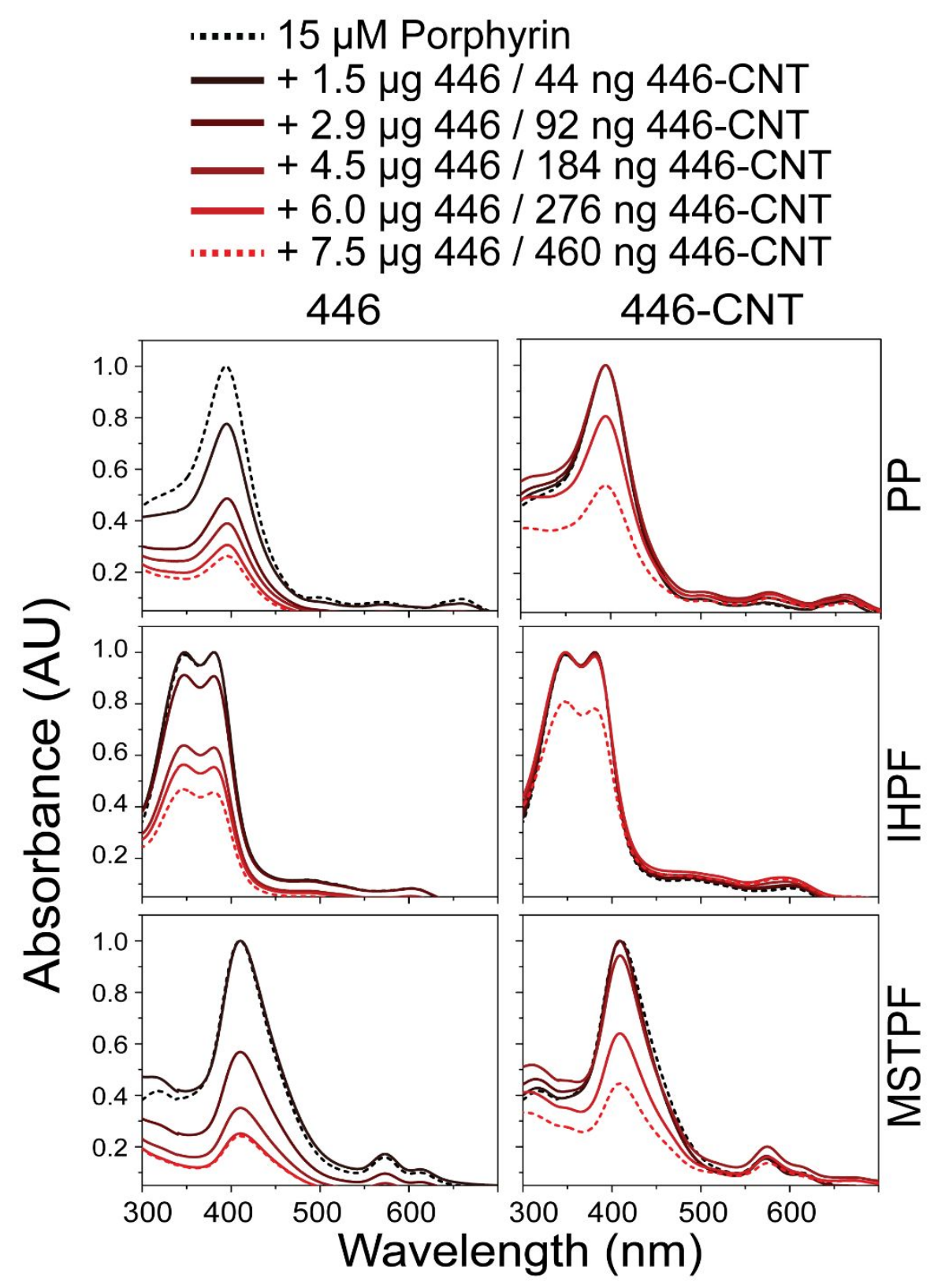

Figure S7 Porphyrin Soret absorption assay. $15 \mu \mathrm{M}$ of the indicated porphyrins were titrated with increasing 446 (left column) or 446-CNT (right column). 

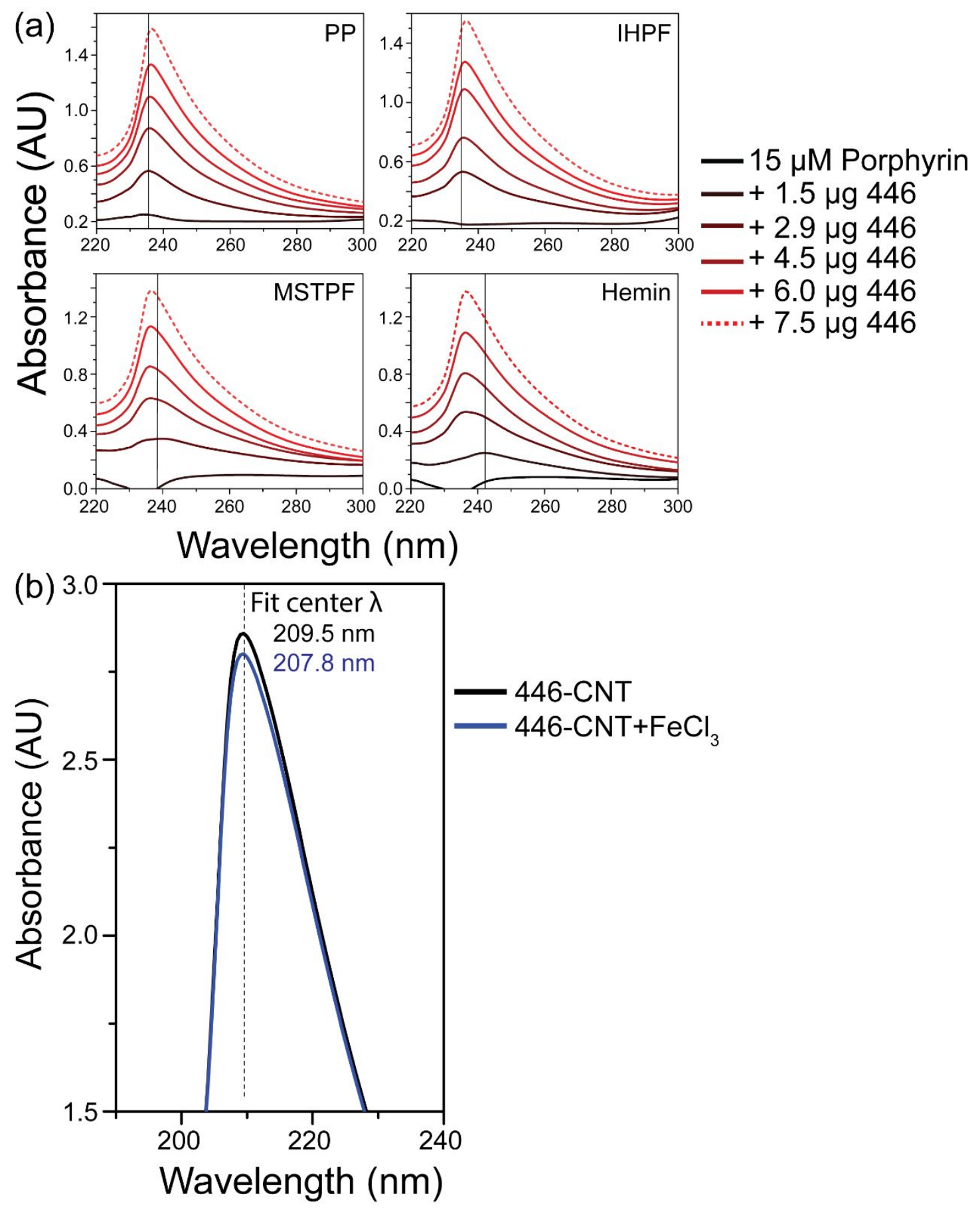

Figure S8 UV absorbance assay of polymer-nanotube-porphyrin interactions. (a) Change of the ultraviolet transition band upon addition of $15 \mu \mathrm{M}$ porphyrin to the indicated amount of polymer 446. Vertical line outlines the polymer-free position. (b) Absorbance spectra of 446-CNT complexes mixed with iron (III) chloride (1 pM) in aqueous buffer. Peak center wavelength values are listed. 
(a)

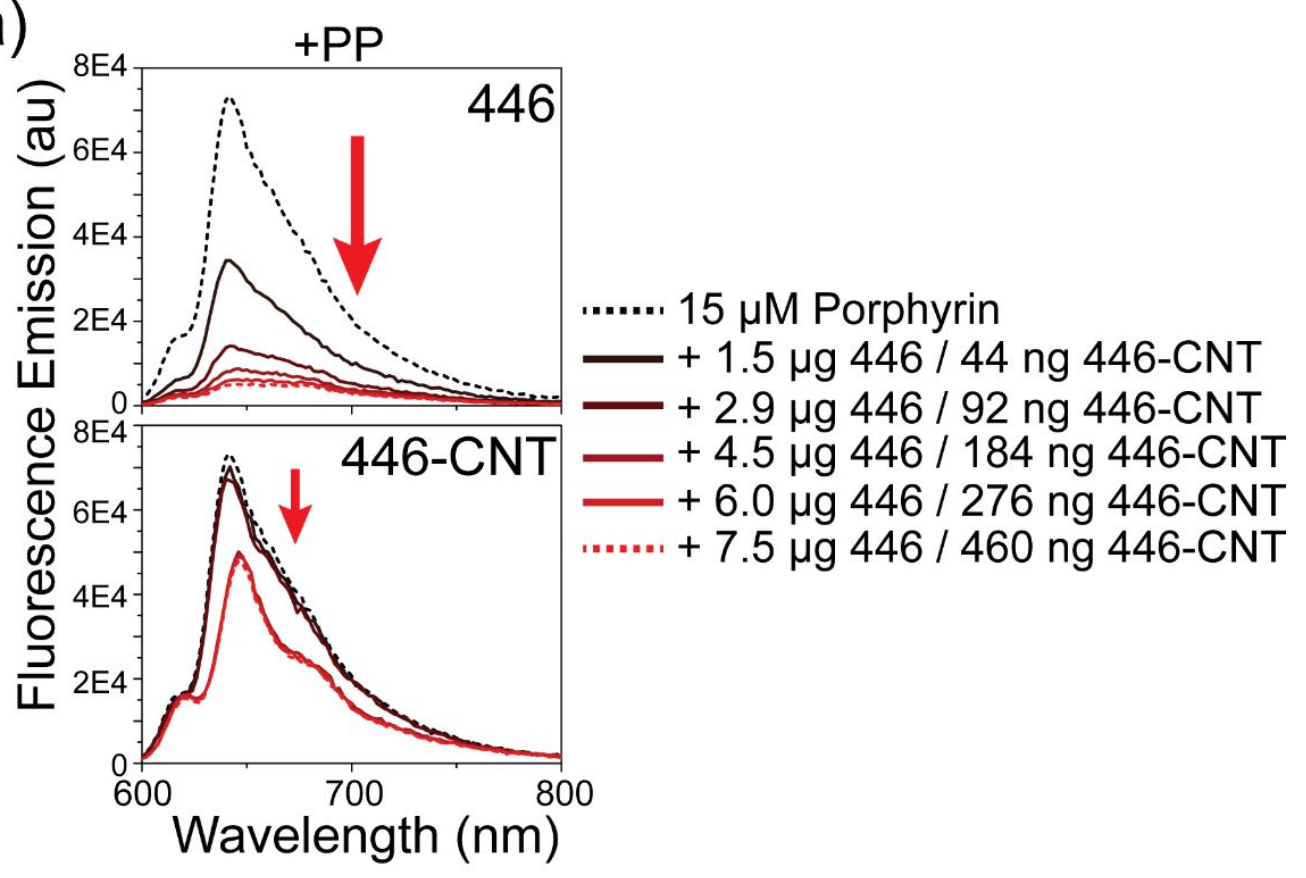

(b)

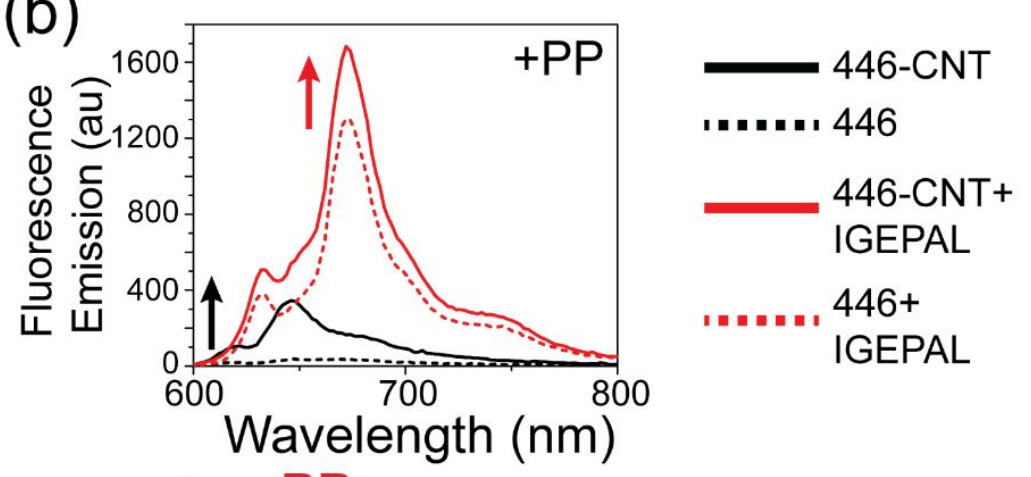

(c)

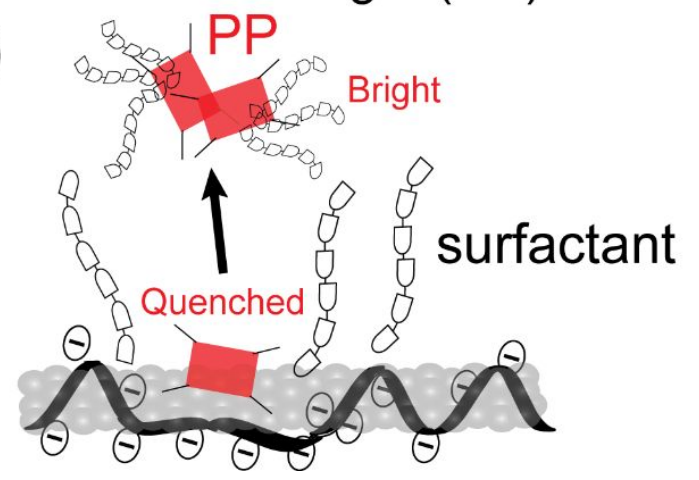

Figure S9 Surfactant displacement assay. (a) Protoporphyrin IX (PP) Soret emission after titrating with polymer 446 (top) or 446-CNT complexes (bottom). Excitation $=$ $380 \mathrm{~nm}$. (b) PP emission from acceptor component samples with or without the nonionic surfactant, IPEGAL CA-630 $(0.05 \% \mathrm{v} / \mathrm{v})$. (c) Model of the expected quenching of dye emission after surfactant displaces the coating and permits binding. 